\title{
Extremity injuries and dementia disproportionately increase the risk for long-term care at older age in an analysis of German Health Insurance routine data for the years 2006 to 2010
}

\author{
Alexander Barth ${ }^{\text {**}}$ (D, Anja Vatterrott ${ }^{1}$, Ying Zhou ${ }^{1}$, Anne Fink ${ }^{2}$ and Gabriele Doblhammer ${ }^{1,3,4}$
}

\begin{abstract}
Background: Extremity injuries (EI) and dementia are important causes of long-term care (LTC), but they can also cause each other and are often present concurrently. Mobility-limiting El can increase the risk of dementia, and dementia increases the risk for falls, which are often the cause of El. When El and dementia are present together, they can increase their negative effect on long-term care risk. This study aims to assess the strength of this interaction and the role of different body regions and severities of El regarding LTC risk.

Methods: We use Cox proportional-hazard models on LTC as dependent variable. El (primarily fractures) and dementia (all types) are the central independent variables. We control for age, sex, rehabilitation and 18 relevant comorbidities. Analyses are based on health claims records for 2004-2010 for a random sample of about 122.000 insurants of Germany's largest public health insurance "AOK" aged 65+, about 25.000 of whom entered LTC.

Results: Without concurrent dementia, non-severe EI (NSEI) of the lower and both extremities and all kinds of severe El (SEI) increase LTC risk (HR: hazard ratio with 95\% confidence interval. Lower NSEl: HR=1.09 [1.05-1.14]; both NSEl: $H R=1.36$ [1.29-1.44]. Lower SEl: $H R=1.67$ [1.57-1.79]; upper SEl: $H R=1.27$ [1.19-1.37]; both $S E l: H R=1.94$ [1.81-2.07]). Dementia alone increases LTC risk more than fourfold (HR $=4.23$ [4.11-4.35]).

Taking the interaction of El and dementia into account, the concurrent presence of El and dementia tends to increase the LTC risk more than expected for lower as well as upper NSEl and SEl. Summarily, when lower or upper $\mathrm{El}$ and dementia are both present, the LTC risk tends to be higher than expected, suggesting synergistic effects.

Conclusions: $\mathrm{El}$ and dementia are important independent risk factors for long-term care. When lower or upper $\mathrm{El}$ and dementia are present together, the resulting long-term care risk is increased disproportionately. Since the concurrent presence of both conditions increases the risk for care need, and a working treatment for dementia is not in sight, preventing El, lessening the impact of El and improving the outlook after an El could help to reduce LTC need in the coming decades.
\end{abstract}

Keywords: Extremities, Mobility limitation, Dementia, Long-term care, Aged

\footnotetext{
*Correspondence: alexander.barth@uni-rostock.de

${ }^{1}$ Empirical Social Research and Demography, Institute of Sociology and

Demography, University of Rostock, Ulmenstr. 69, 18057 Rostock, Germany

Full list of author information is available at the end of the article
} 


\section{Key points}

Dementia and extremity injuries can increase each other's impact on long-term care risk. The effect of dementia and simultaneous extremity injury increases the long-term care risk beyond that caused by dementia alone and more than expected, suggesting synergistic effects. The size of the combined risk depends on the body region and severity of the extremity injury. Severe extremity injury increases risk more than upper extremity injury, and lower extremity injury more than upper extremity injury. Targeted prevention and treatment of extremity injuries might help to reduce long-term care need in the future, even if a treatment for dementia is not forthcoming.

\section{Background}

Extremity injuries (EI) due to falls [1,2] are common among older persons [3]. Together with dementia [4], they are important causes of long term care (LTC) [5-8]. In western societies older age groups are growing due to rising life expectancy and large cohorts entering old age. Older people are more likely to experience falls and subsequently incur fractures than younger people [9] and to experience dementia. Increasing EI incidence has also been noted due to increasing fracture incidence independent of age structure, most likely because the number and severity of falls in older populations has increased [10]. In the future, even more people may be expected to experience and live longer with dementia, thus both risk factors might become even more important [11]. EI and dementia will continue to affect LTC need - a field already under considerable strain due to financial and personnel shortages. Not much is known about the combined effects of EI and dementia on LTC, especially in terms of a finer distinction of severe and non-severe injuries of the lower, upper or both extremities. EI and dementia are discrete LTC risks, but can also be causally related and are often present together [12-14].

Maintaining an active and regular social life as well as regularly performing productive tasks decreases the risk of dementia [15], because the associated cognitive stimulation helps preserve cognitive functioning. Lower and upper EI can drastically reduce the ability to uphold social interaction or perform productive tasks at the usual levels and act as a risk factor for dementia in that they reduce cognitive stimulation $[16,17]$. Lower EI that limit mobility might be even more influential. Thus, the onset of mobility problems, such as unsteady gait, is recognized as a valid predictor of later cognitive decline $[14,18,19]$. An EI can also indirectly increase the risk for dementia due to a subsequent delirium, which is a risk factor for dementia as well [20].

Conversely, dementia is a pre-existing condition for many fractures, because cognitive deterioration also affects gait and balance, increasing the risk of falling $[9,14,21,22]$. Dementia thus leads to earlier or more frequent falls and may also increase the severity of fallrelated injuries. If present concurrently, the complementary effect of the two central LTC risk factors EI and dementia might further compound the LTC risk. However, the strength of their synergistic effect and to what degree the body region and severity of an EI influence the resulting LTC risk remains unclear.

Our study addresses four topics. First we differentiate between severe and non-severe injuries and hypothesize that Severe EI (SEI) leads to higher LTC risk than NonSevere EI (NSEI). Second, we distinguish between lower, upper, and both extremities. Lower EI affect movement, upper EI affect manipulation of the environment, and a combination of both might differently affect LTC risk on their own or in conjunction with dementia. Moreover, both kinds of EI may indicate either one drastic injury that affects both extremities or multiple incidents. Third, as limited mobility may reduce cognitive activity and mobility, mobility-limiting lower EI and dementia should increase LTC risk more than upper EI and dementia, with SEI generally causing a higher risk than NSEI. Fourth, we explore the combined effects of dementia and EI on LTC, expecting them to be larger than the individual effect sizes simply added up [23].

\section{Methods}

Data

We use health claims data covering a random sample $(N=250.000)$ of insurants from ages 50 and higher of Germany's largest public health insurance, AOK ("Allgemeine Ortskrankenkasse"). AOK covers one in three citizens aged $50+$, with an even higher share at older ages where it reaches 50\% among the oldest old. As health insurance is mandatory, only few citizens (less than $0.3 \%$ ) are not covered by any health insurance, $11 \%$ of the population is covered by private health insurance. Over all ages combined, the AOK sample differs from the German population in terms of age structure and socio-economic status. However, the differences become small at the highest ages where dementia is most prominent. Additionally, the age-specific death rates from our data indicate that mortality in AOK data fits well with the mortality of the whole German population, as another study using the same data shows [24]. An age-stratified sample was drawn based on all insurants in the first quarter of 2004 who were born in or before 1954, regardless of seeking treatment. Longitudinal observation takes place from 2004 to 2010 and contains one spell per quarter. The study period covers 2006-2010. The two preceding years are used to verify dementia diagnoses by means of multiple diagnoses by different specialists or over time. Thus all prevalent dementia diagnoses from 2006 onwards are validated. 
Claims data are process-generated, detailed, and comprehensive. They contain all inpatient and outpatient diagnoses as ICD-10-codes, diagnosing specialist, prescriptions, rehabilitations with prognosis, costs, dates of birth and death, and sex. Only those diagnoses relevant for treatment and refundable by the insurer are recorded. All population groups are covered, including groups such as institutionalized persons in LTC, who are often subject to EI or dementia and missing in other studies. Although common survey problems such as selectivity or recall uncertainty are avoided, variables such as socioeconomic status or the severity of dementia are not available.

\section{Case count and variables}

Cleaning, consistency checking, and especially the reduction to those at least 65 years old left 156.527 persons/770.000 person-years. As our outcome variable is the incidence of LTC, we removed all persons who were already in LTC in 2004-2005.

\section{Dependent variable: long term care entry}

We define individuals who received benefits from Germany's statutory long-term care insurance as being in need of LTC. The LTC insurance ("Pflegeversicherung") was established in 1995 as a pay-as-you-go scheme, just like other branches of Germany's social security system (e.g. health care, unemployment). LTC benefits are granted when an applicant passes an objective assessment primarily focused on ADL impairments and is assigned to one of three LTC levels that denote the amount of care required. The lowest level is granted when at least one and a half hours of care are required per day, at least half of which concern basic tasks. The highest level is granted when at least five hours of care are required (four of which concerning basic tasks). In our study, we do not distinguish the different LTC levels. The LTC variable is binary; it has and retains the value one from the time any level of LTC is first acquired.

\section{Independent variables: extremity injuries, dementia and comorbidities}

EI are differentiated by body region and severity using diagnostic data of fractures and injuries of the upper and lower extremities, including the pelvis (ICD-10 codes S40-S99, relevant parts of T for wounds, luxations, contusions, burns, frostbites and amputations). Rehabilitation data allow us to identify severe EI cases, which are cases of EI who concurrently or later received a medium or long term rehabilitative measure prescribed specifically due to constraints in relevant activities of daily living or multistructural functional damages related to EI. We do not distinguish between different methods of rehabilitation and rehabilitative measures prescribed due to other conditions than EI are not considered. This allows for the differentiation of six EI types: Non-Severe Extremity Injuries (NSEI), which are classified as (1) lower NSEI for fractures and injuries from the foot up to the hip and also the pelvis, (2) upper NSEI for fractures in the hands, arms and shoulders, and (3) lower and upper NSEI when both lower and upper extremities are affected); and Severe Extremity Injuries (SEI) of the (4) lower, (5) upper, or (6) both extremities. Multiple injuries of the same extremity over time are not considered separately. Pelvic injuries are included as lower extremity injuries because they are common fall-related injuries and are comparable to other lower limb injuries in terms of their consequences [25].

Dementia is defined as one or more of the following diagnoses: Alzheimer's disease (ICD-10 codes F00/G30), vascular dementia (F01), Lewy body dementia (G31.82), circumscribed brain atrophy (G31.0), dementia as a sideeffect of another disease, e.g. Parkinson's disease (F02, F05.1, G23.1), as well as other/not specified dementia (F03). To ensure validity, we considered only diagnoses flagged as "secure diagnosis" for outpatients or "discharge diagnosis" for inpatients. Second, at least two concurrent or later diagnoses either by an inpatient and an outpatient physician or from different outpatient specialists were required. If the first dementia diagnosis was given in the last quarter of the observation period, it was deemed valid.

Age is a categorical variable in 5 year-groups starting at ages 65-69, with the last age category 95 years and older. Chronic diseases and other ailments are included as binary variables taking the value one from the first occurrence from 2004 onwards and zero otherwise. They are: hypertension (relevant parts of I10-I15), diabetes (E10-E14), ischemic diseases (I20-I25), cerebral diseases (I60-69), hypercholesterolemia (E780), atrial fibrillation (I48), heart insufficiency (I50), lung insufficiency (J44), nervous diseases except Parkinson's disease and dementia (G20-G22), Parkinson's disease (G20-G22), gastric diseases (K0-K9), alcoholic liver disease (K70), atherosclerosis (I70), pneumonia (J12-J18), cases of infections or parasites (A-B), other external injuries ( $\mathrm{S}-\mathrm{T}, \mathrm{V}-\mathrm{Y}$ except related to EI), and smoking as well as non-smoking related cancers. All variables and corresponding occurrences, person-year exposures, and transition rates into LTC are shown in Table 1.

\section{Statistical analysis}

We use Kaplan-Meier survival analysis and Cox proportional hazard models to study LTC risk depending on EI and dementia, adjusted for comorbidities and other controls. Calendar time of the study period (2006-2010) is used as underlying process time. Diagnoses and other status changes (except death, which is given by the 
Table 1 Sample overview of long term care entry cases by age, sex, extremity injuries, rehabilitation, dementia and comorbidities

\begin{tabular}{|c|c|c|c|c|c|}
\hline & Exposure (person years) & Cases & Incidence rate p. $100 \mathrm{PY}$ & $95 \%$ & \\
\hline Total & 510,957 & 25,150 & 4.9 & 4.9 & 5.0 \\
\hline \multicolumn{6}{|l|}{ Age groups } \\
\hline $65-69$ & 93,189 & 1213 & 1.3 & 1.2 & 1.4 \\
\hline $70-74$ & 167,840 & 3641 & 2.2 & 2.1 & 2.2 \\
\hline $75-79$ & 125,332 & 5242 & 4.2 & 4.1 & 4.3 \\
\hline $80-84$ & 80,730 & 6781 & 8.4 & 8.2 & 8.6 \\
\hline $85-89$ & 34,889 & 5584 & 16.0 & 15.6 & 16.4 \\
\hline $90-94$ & 7485 & 2090 & 27.9 & 26.8 & 29.1 \\
\hline $95+$ & 1491 & 599 & 40.2 & 37.1 & 43.5 \\
\hline \multicolumn{6}{|l|}{ Sex } \\
\hline Male & 200,175 & 8592 & 4.3 & 4.2 & 4.4 \\
\hline Female & 310,782 & 16,558 & 5.3 & 5.2 & 5.4 \\
\hline No El & 368,033 & 16,058 & 4.4 & 4.3 & 4.4 \\
\hline Lower NSEI & 48,770 & 2781 & 5.7 & 5.5 & 5.9 \\
\hline Upper NSEI & 41,947 & 2130 & 5.1 & 4.9 & 5.3 \\
\hline Lower \& upper NSEI & 17,286 & 1455 & 8.4 & 8.0 & 8.9 \\
\hline Lower SEl & 12,324 & 972 & 7.9 & 7.4 & 8.4 \\
\hline Upper SEl & 13,677 & 816 & 6.0 & 5.6 & 6.4 \\
\hline Lower \& upper SEl & 8920 & 938 & 10.5 & 9.9 & 11.2 \\
\hline Rehabilitation & 451,824 & 20,268 & 4.5 & 4.4 & 4.5 \\
\hline Yes & 59,133 & 4882 & 8.3 & 8.0 & 8.5 \\
\hline Dementia & 484,204 & 17,939 & 3.7 & 3.7 & 3.8 \\
\hline Yes & 26,753 & 7211 & 27.0 & 26.3 & 27.6 \\
\hline Hypertension & 102,676 & 6103 & 5.9 & 5.8 & 6.1 \\
\hline Yes & 408,281 & 19,047 & 4.7 & 4.6 & 4.7 \\
\hline Diabetes & 336,051 & 15,158 & 4.4 & 4.4 & 4.6 \\
\hline Yes & 174,905 & 9992 & 5.7 & 5.6 & 5.8 \\
\hline Ischemic stroke & 308,858 & 13,257 & 4.3 & 4.2 & 4.4 \\
\hline Yes & 202,098 & 11,893 & 5.9 & 5.8 & 6.0 \\
\hline Cerebral diseases & 398,693 & 15,270 & 3.8 & 3.8 & 3.9 \\
\hline Yes & 112,264 & 9880 & 8.8 & 8.6 & 9.0 \\
\hline Hypercholesterolemia & 347,585 & 18,762 & 5.4 & 5.3 & 5.5 \\
\hline Yes & 163,372 & 6388 & 3.9 & 3.8 & 4.0 \\
\hline Atrial fibrillation & 442,155 & 18,481 & 4.2 & 4.1 & 4.2 \\
\hline Yes & 68,801 & 6669 & 9.7 & 9.5 & 9.9 \\
\hline Heart insufficiency & 391,477 & 13,800 & 3.5 & 3.5 & 3.6 \\
\hline Yes & 119,480 & 11,350 & 9.5 & 9.3 & 9.7 \\
\hline Lung insufficiency & 429,475 & 20,109 & 4.7 & 4.6 & 4.7 \\
\hline Yes & 81,482 & 5041 & 6.2 & 6.0 & 6.4 \\
\hline Nervous diseases & 288,980 & 11,675 & 4.0 & 4.0 & 4.1 \\
\hline Yes & 221,976 & 13,475 & 6.1 & 6.0 & 6.2 \\
\hline Parkinson's disease & 500,037 & 23,519 & 4.7 & 4.6 & 4.8 \\
\hline Yes & 10,919 & 1631 & 15.0 & 14.2 & 15.7 \\
\hline Gastric diseases & 181,926 & 8846 & 4.9 & 4.8 & 5.0 \\
\hline
\end{tabular}


Table 1 Sample overview of long term care entry cases by age, sex, extremity injuries, rehabilitation, dementia and comorbidities (Continued)

\begin{tabular}{|c|c|c|c|c|c|}
\hline Yes & 329,031 & 16,304 & 5.0 & 4.9 & 5.0 \\
\hline Alcoholic liver disease & 507,071 & 24,855 & 4.9 & 4.8 & 5.0 \\
\hline Yes & 3886 & 295 & 7.6 & 6.8 & 8.5 \\
\hline Atherosclerosis & 434,115 & 19,991 & 4.6 & 4.5 & 4.7 \\
\hline Yes & 76,841 & 5159 & 6.7 & 6.5 & 6.9 \\
\hline Pneumonia & 477,083 & 20,980 & 4.4 & 4.3 & 4.5 \\
\hline Yes & 33,874 & 4170 & 12.3 & 11.9 & 12.7 \\
\hline Infections/Parasites & 282,266 & 11,997 & 4.3 & 4.2 & 4.3 \\
\hline Yes & 228,690 & 13,153 & 5.8 & 5.7 & 5.9 \\
\hline External injuries & 254,719 & 10,546 & 4.1 & 4.1 & 4.2 \\
\hline Yes & 256,238 & 14,604 & 5.7 & 5.6 & 5.8 \\
\hline Smoking-related cancer & 472,757 & 21,559 & 4.6 & 4.5 & 4.6 \\
\hline Yes & 38,199 & 3591 & 9.4 & 9.1 & 9.7 \\
\hline Non-smoking r. cancer & 462,228 & 21,415 & 4.6 & 4.6 & 4.7 \\
\hline Yes & 48,729 & 3735 & 7.7 & 7.4 & 7.9 \\
\hline
\end{tabular}

PY person years, $\mathrm{Cl}$ confidence interval

Source: AOK claims data, own calculations

month of death) are placed in the middle of the quarter in which they occur. If death and care entry occur in the same quarter, care entry was placed in the month before death. LTC entry is available quarterly, with the exception of 2006, where it is available only at the end of the year and was placed mid-year. All diagnoses, age group and care-level entry are treated as time dependent, sex is time constant. All medical diagnoses, including EI and dementia, indicate whether a certain disease or status was ever observed starting from 2004 onwards. They were coded as one and remained like that from that point onwards until the end of the observation period. Thus, they can be interpreted as the effect of ever having experienced the respective condition since the beginning of 2004. Individuals are followed until censoring or death, depending on which occurred first.

\section{Results}

Within the period of study 25,150 individuals received a care level, which is an incidence rate of $4.9(95 \% \mathrm{CI}=$ 4.9-5.0) individuals per 100 person years (Table 1). Dementia patients have an incidence rate seven times higher than non-demented individuals, which makes it the strongest cause of LTC after age higher than 90 years. Individuals without EI have a lower incidence rate than patients with any kind of EI. Generally, NSEI patients have lower incidence rates than SEI patients, and patients with upper EI have lower incidence rates than patients with lower or both types of EI. Patients with severe upper and lower EI show a relative risk nearly 2.5 times higher than patients without EI. The transition into LTC increases with age; the incidence rate roughly doubles for each 5 year interval. Females have a slightly higher incidence rate than males, and patients who received rehabilitative treatment show nearly double the incidence rate of those who did not, indicating that these treatments are typically reserved for more serious cases.

Dementia leads to faster LTC entry (Fig. 1a), as does EI. In particular, LTC risk increases from no to non-severe to severe EI, whereby upper EI generally show lower risk than lower EI, and both EI show higher risk than upper or lower EI. There is one exception, namely upper SEI, which increase the LTC risk less than both NSEI (Fig. 1b). Without dementia, SEI is an important risk factor for LTC, whereas NSEI does not differ from no EI (Fig. 1c). Compared to dementia alone, NSEI and SEI with dementia are associated with faster LTC transition. The body region affected by an EI plays an important role for LTC transition. Without dementia, especially both and lower EI increase LTC transition compared to upper and no EI. Compared to no EI with dementia, both EI and, to a smaller extent, lower and upper EI, increase the LTC transition (Fig. 1d). Log-rank and Wilcoxon tests generally indicate highly significant differences between the survivor functions.

Hazard ratios estimated by multivariate Cox proportional hazard models are presented in Table 2. The first model shows the main effects of EI, dementia, age and sex. The second model presents the EI-dementia interaction. Comorbidities and rehabilitation are added in model 3.

\section{Extremity injuries}

For all body regions, SEI increase LTC risk more than NSEI (Table 2, Model 1), e.g. lower SEI are associated with a hazard ratio (HR) of 1.67 (95\% CI: 1.57-1.79) and lower 

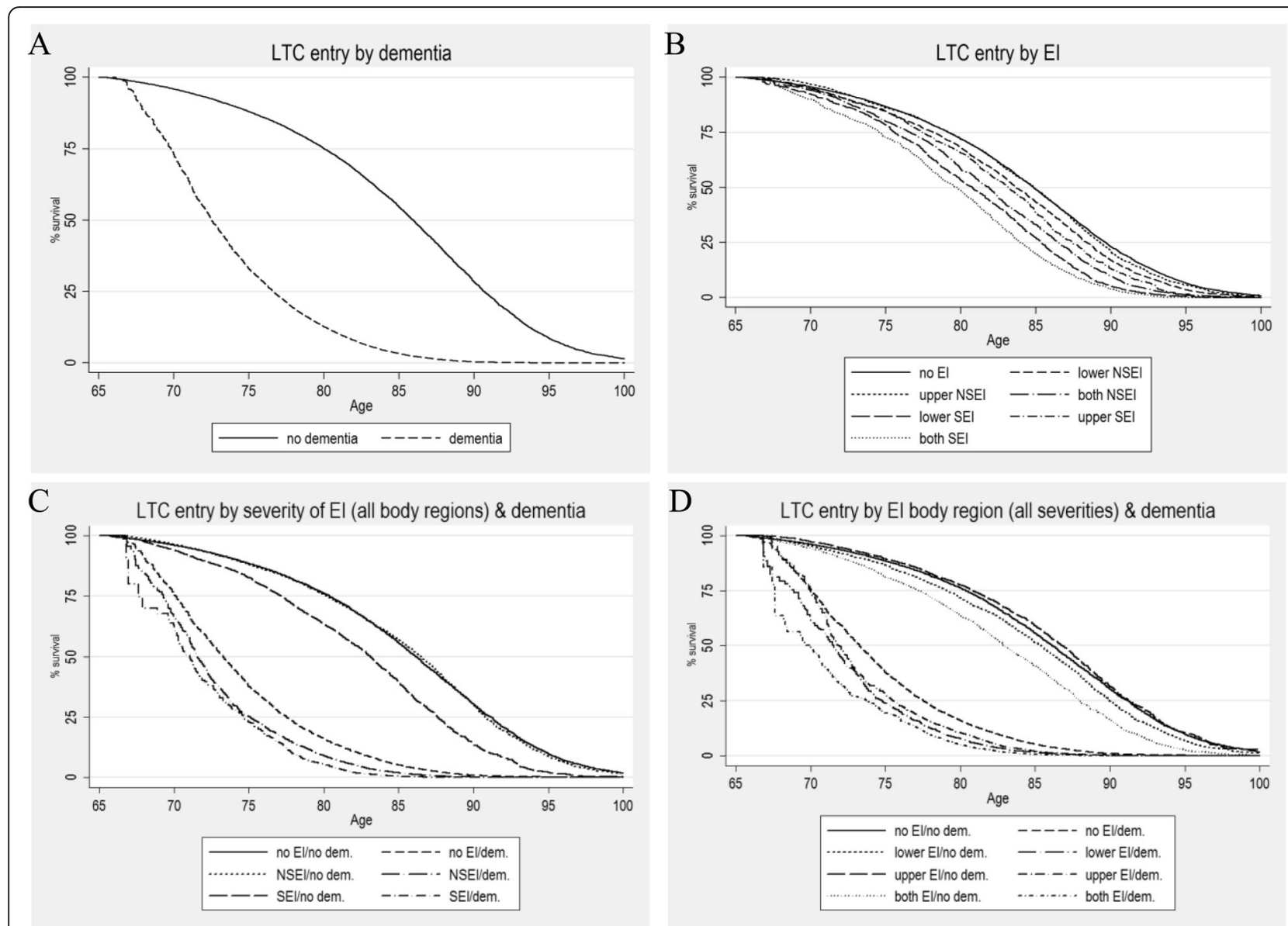

Fig. 1 a-d Long term care entry over age by extremity injuries and dementia (Kaplan-Meier Survival Curves). Source: AOK claims data, own calculations

NSEI with a HR of 1.09 (95\% CI: 1.05-1.14). Both EI together cause a higher LTC risk than EI of only one body region for NSEI (HR $=1.36,95 \% \mathrm{CI}: 1.29-1.44)$ and SEI $(\mathrm{HR}=1.94,95 \%$ CI: $1.81-2.07)$. Upper NSEI show no significant effect on LTC risk. Dementia is a distinct and strong LTC risk factor $(\mathrm{HR}=4.23,95 \%$ CI: 4.11-4.35).

\section{Extremity injuries with concurrent dementia}

To assess the interaction between EI and dementia, the observed joint effects (Model 2) are compared with the expected joint effects (based on Model 1). The LTC risk resulting from the concurrent presence of EI and dementia tends to be greater than the expected risk resulting from lower and upper NSEI as well as SEI, and slightly smaller than expected for NSEI and SEI of both extremities at once. For instance, the expected joint effect of upper NSEI with concurrent dementia is $\mathrm{HR}=4.1$ ( $\mathrm{HR}$ dementia * $\mathrm{HR}$ upper NSEI: $4.23 * 0.97$ ), while the observed joint effect is $H R=4.74$. Thus, for NSEI as well as SEI, the concurrent presence of dementia affects the LTC risk resulting from lower or upper EI, while it does not increase the LTC risk when both upper and lower extremities are injured.

Without concurrent dementia, the same pattern as in model 1 emerges regarding the role of EI severity. SEI of a given body region show higher LTC risk than NSEI in the same region, e.g. lower SEI with HR $=1.63$ (95\% CI: 1.501.76) and lower NSEI with HR $=1.03$ (95\% CI: 0.98-1.09, not significant). Interestingly, upper NSEI without dementia even show a protective effect $(\mathrm{HR}=0.86,95 \%$ CI: $0.81-$ 0.91 ), but when dementia is present concurrently, LTC risk is increased, as the resulting joint effect $(\mathrm{HR}=4.74$, 95\% CI: 4.42-5.09) is higher than that of dementia alone.

Dementia alone remains an important LTC risk factor, as it increases LTC risk nearly fourfold ( $\mathrm{HR}=3.92,95 \%$ CI: 3.77-4.07). Summarily, LTC risk is higher for all kinds of EI if dementia is present as well, and also tends to be higher than expected in the cases of lower or upper NSEI and SEI.

\section{Full model including comorbidities}

Finally, adding comorbidities (see Appendix) and rehabilitation reduces the effect sizes of EI and dementia, 
Table 2 Hazard ratios of long term care entry by age, sex, extremity injuries, rehabilitation and dementia

\begin{tabular}{|c|c|c|c|c|c|c|c|c|c|c|}
\hline & \multicolumn{3}{|c|}{ Model $1^{\text {a }}$} & & \multicolumn{3}{|c|}{ Model $2^{b}$} & \multicolumn{3}{|c|}{ Model $3^{c}$} \\
\hline & $\overline{\mathrm{HR}}$ & \multicolumn{2}{|l|}{$95 \% \mathrm{Cl}$} & & $\overline{\mathrm{HR}}$ & \multicolumn{2}{|l|}{$95 \% \mathrm{Cl}$} & $\overline{\mathrm{HR}}$ & \multicolumn{2}{|l|}{$95 \% \mathrm{Cl}$} \\
\hline \multicolumn{11}{|l|}{ Age } \\
\hline 65-69 (ref.) & 1.00 & & & & 1.00 & & & 1.00 & & \\
\hline $70-74$ & 1.62 & 1.51 & 1.73 & & 1.62 & 1.52 & 1.73 & 1.56 & 1.47 & 1.67 \\
\hline $75-79$ & 2.89 & 2.72 & 3.08 & & 2.90 & 2.72 & 3.09 & 2.65 & 2.49 & 2.83 \\
\hline $80-84$ & 5.25 & 4.93 & 5.58 & & 5.26 & 4.95 & 5.60 & 4.53 & 4.26 & 4.83 \\
\hline $85-89$ & 8.96 & 8.41 & 9.55 & & 8.99 & 8.43 & 9.58 & 7.51 & 7.04 & 8.01 \\
\hline $90-94$ & 14.29 & 13.30 & 15.36 & & 14.32 & 13.32 & 15.39 & 11.51 & 10.69 & 12.39 \\
\hline 95 plus & 18.91 & 17.12 & 20.89 & & 18.87 & 17.08 & 20.84 & 15.46 & 13.98 & 17.10 \\
\hline Male (ref.) & 1.00 & & & & 1.00 & & & 1.00 & & \\
\hline Female & 0.90 & 0.88 & 0.92 & & 0.90 & 0.88 & 0.92 & 1.05 & 1.02 & 1.08 \\
\hline No rehabilitation (ref.) & & & & & & & & 1.00 & & \\
\hline Rehabilitation & & & & & & & & 1.50 & 1.45 & 1.55 \\
\hline No dementia & 1.00 & & & & & & & & & \\
\hline Dementia & 4.23 & 4.11 & 4.35 & & & & & & & \\
\hline \multicolumn{11}{|l|}{ No El } \\
\hline & 1.00 & & & No dementia & 1.00 & & & 1.00 & & \\
\hline & & & & Dementia & 3.92 & 3.77 & 4.07 & 3.30 & 3.17 & 3.43 \\
\hline \multicolumn{11}{|l|}{ NSEI } \\
\hline \multirow[t]{2}{*}{ Lower } & 1.09 & 1.05 & 1.14 & No dementia & 1.03 & 0.98 & 1.09 & 0.98 & 0.93 & 1.03 \\
\hline & & & & Dementia & 4.87 & 4.57 & 5.19 & 3.91 & 3.66 & 4.17 \\
\hline \multirow[t]{2}{*}{ Upper } & 0.97 & 0.92 & 1.01 & No dementia & 0.86 & 0.81 & 0.91 & 0.83 & 0.78 & 0.88 \\
\hline & & & & Dementia & 4.74 & 4.42 & 5.09 & 3.71 & 3.46 & 3.99 \\
\hline \multirow[t]{2}{*}{ Both } & 1.36 & 1.29 & 1.44 & No dementia & 1.34 & 1.25 & 1.43 & 1.22 & 1.14 & 1.31 \\
\hline & & & & Dementia & 5.67 & 5.22 & 6.16 & 4.23 & 3.89 & 4.60 \\
\hline \multicolumn{11}{|l|}{ SEI } \\
\hline \multirow[t]{2}{*}{ Lower } & 1.67 & 1.57 & 1.79 & No dementia & 1.63 & 1.50 & 1.76 & 1.35 & 1.25 & 1.46 \\
\hline & & & & Dementia & 7.08 & 6.33 & 7.92 & 5.00 & 4.47 & 5.60 \\
\hline \multirow[t]{2}{*}{ Upper } & 1.27 & 1.19 & 1.37 & No dementia & 1.19 & 1.09 & 1.29 & 1.11 & 1.02 & 1.21 \\
\hline & & & & Dementia & 5.97 & 5.26 & 6.77 & 4.85 & 4.27 & 5.51 \\
\hline \multirow[t]{2}{*}{ Both } & 1.94 & 1.81 & 2.07 & No dementia & 1.95 & 1.79 & 2.11 & 1.63 & 1.50 & 1.77 \\
\hline & & & & Dementia & 7.73 & 6.92 & 8.63 & 5.06 & 4.52 & 5.66 \\
\hline
\end{tabular}

${ }^{a}$ Model 1 includes extremity injuries and dementia as individual variables and no interaction effect

${ }^{\mathrm{b}}$ Models 2 and 3 include extremity injuries and dementia as interaction effect

cModel 3 controls for comorbidities (see Appendix for multivariate results)

Source: AOK claims data, own calculations

but the relation of different kinds and severities of EI and dementia to each other that emerged in the previous models remains unchanged (Model 3). For example, lower SEI with dementia (HR $=5.00,95 \%$ CI: 4.47-5.60) show a higher relative risk than lower NSEI with dementia $(\mathrm{HR}=3.91,95 \% \mathrm{CI}: 3.66-4.17)$. The comorbidities show mostly higher LTC risk, with the exceptions of gastric diseases, ischemic stroke, hypertension, and hypercholesterolemia. Rehabilitative treatment is associated with higher LTC risk (HR $=1.50,95 \% \mathrm{CI}$ : $1.45-1.55)$ and seems to be prescribed to people who are more likely to end up in LTC.

\section{Discussion}

We show that concurrent with dementia, NSEI and SEI increase the LTC risk above the level associated with dementia alone. Moreover, when concurrent with dementia, upper or lower non-severe and severe EI tend to show LTC risks that are larger than expected. Because both afflictions are common comorbidities that can 
influence or strengthen each other, this fits in with previous work. Even without such interaction, EI and dementia are two central independent LTC risk factors. While confirming the importance of dementia on LTC, we also show that SEI increase LTC risk more than NSEI, and among both degrees of severity, upper EI contribute less risk than lower or both SEI. EI can severely limit basic self care capabilities, e.g. transfer within the home [26], hygiene, or food preparation, which are qualifiers for LTC benefits. Only lower SEI, which could affect transfer within the home, show increased LTC risk. Moving to where a specific task should take place is a necessary prerequisite of performing said task, thus EI limiting mobility can be seen as a cause for LTC on a more basic level than upper EI limiting interaction. LTC risk linked to upper EI is a significant influence only when the EI is severe, or when concurrent with dementia. Dementia can, depending on its progress, necessitate LTC because it constrains the ability to live independently on many levels [5, 7]. Lower EI are not only problematic in itself, as our results show, but are also a prominent cause for decline in physical activity, which has been linked to a higher occurrence of dementia $[12,13]$. Lower EI can impede social interaction and, by extension, cognitive activity $[18,19]$. Dementia is often a pre-existing condition for falls and fractures [9], thus dementia patients sustain EI more often than those free of dementia. Keeping a balanced gait relies on the same brain area affected by dementia, thus explaining why, at older ages, mobility-limiting EI can often be traced back to cognitive deficits $[21,22]$. This mutual influence might explain why individuals with dementia and EI show the highest LTC risk.

\section{Strengths and weaknesses}

This study's main weaknesses are rooted in the data, which were collected in conjunction with the refunding of medical practitioners. Thus, dementia might be underreported, because only diagnoses related to an actual treatment are recorded. Thus, early stage dementia or severe dementia might not be documented at all times, because a treatment is not yet deemed reasonable or futile [11]. If dementia was only diagnosed once during the study time, it would not register as such in our data due to our reliance on the validation procedure that requires at least two diagnoses. Second, we chose to define EI in a strict sense. Other injuries that might be comparable to EI in terms of effects on mobility limitation are thus not classified as EI. Furthermore, not every SEI can be classified as such, because we rely on rehabilitation data, and available funds for rehabilitative treatments are limited. Thus, not every SEI case might receive such treatment. Only diagnoses relevant for treatment and refundable by the insurer are recorded.
The definition of LTC is based solely on whether a person received benefits from the statutory LTC insurance. Not everyone in need of care might be classified as such in the data, because someone might not know about the availability of those benefits, or because the application process was deemed too difficult. Last, there might be some selectivity in the choice of private vs. public (such as AOK) health insurance and within the public sector. Even though AOK is Germany's largest public health insurance, our sample is not completely representative of adults older than 49 years, however, with age, the differences become smaller and the mortality in AOK data fits well with the mortality pattern of the whole German population [24].

The main strengths of this study are the claims data used. They are process-generated, detailed, and comprehensive. The outcome variable LTC benefit eligibility is diagnosed externally and objectively. The data are not subject to health-related self-selection beyond the choice of health insurance, because all people insured with AOK older than 50 in the first quarter of 2004 were eligible to be drawn into our sample. The data includes groups such as institutionalized persons in LTC that are often subject to EI or dementia. Other common survey problems, e.g. recall uncertainty, are avoided as well.

\section{Conclusions}

Despite its' importance as a risk factor for care need, the interaction of EI and dementia has not been the explicit subject of many studies. Preventing EI can reduce LTC in two ways: for persons affected only by EI, and for persons in whom EI and concurrent dementia increase LTC risk beyond that associated with dementia alone. As a viable treatment for dementia is not expected to be forthcoming in the foreseeable future and as EI and dementia often occur together, preventing EI or improving their treatment could be a promising strategy to counter LTC need in ageing societies. Because mobility limitations have also been shown to cause or accelerate the onset of dementia [14], better EI prevention and rehabilitation may not only reduce the direct LTC risk, but may also help to prevent dementia. Special attention can be paid to exercises that promote regular activity and which train gait and balance, because they can help prevent falls and subsequent injuries in the first place, but evidence on their effectiveness is mixed and suggests that especially for frail people, exercise alone does not protect from falls, so focusing on the safety of mobility is of high importance. Otherwise, frail people might have a higher risk of falling with higher levels of mobility [27-29]. Even for dementia patients, multifactorial fall prevention programs [30] as well as cognitive-motor dual task exercises for individuals 
without cognitive impairments have been shown to be effective [31]. Innovative mobility-related assistive devices used primarily by older individuals already affected by ML provide another possibility [32]. Needdriven usage of mobility-related assistive devices is the typical case [33], suggesting the main objective should be offering adequate, user-friendly and effective devices, and their adoption by users should follow naturally. The introduction of such devices could even by financed by private copayments because, to a certain extent, there is willingness to effect individual copayments to purchase assistive devices [34]. The rehabilitative process also leaves room for improvements especially tailored for patients with cognitive impairments and EI. For instance, special post-operative, outpatient treatments for dementia patients with hip fractures by specialized personnel effectively helped cognitively impaired fracture patients to regain their pre-injury functional status [35]. If such a support chain were standard, better recovery of previous mobility status could be achieved, thereby reducing or postponing care risk.

\section{Appendix}

Table 3 Hazard ratios of care need by comorbidities

\begin{tabular}{|c|c|c|c|}
\hline & \multicolumn{3}{|c|}{ Model 3* } \\
\hline & $\mathrm{HR}$ & $95 \% \mathrm{Cl}$ & \\
\hline \multicolumn{4}{|l|}{ Diseases (ref: not afflicted) } \\
\hline Hypertension & 0.51 & 0.49 & 0.52 \\
\hline Diabetes & 1.15 & 1.12 & 1.18 \\
\hline Ischemic stroke & 0.83 & 0.80 & 0.85 \\
\hline Cerebral stroke & 1.42 & 1.38 & 1.46 \\
\hline Hypercholesterolemia & 0.75 & 0.73 & 0.78 \\
\hline Atrial fibrillation & 1.41 & 1.37 & 1.45 \\
\hline Parkinson's disease & 1.56 & 1.49 & 1.65 \\
\hline Heart insufficiency & 1.50 & 1.46 & 1.55 \\
\hline Pneumonia & 1.80 & 1.74 & 1.87 \\
\hline Lung insufficiency & 1.04 & 1.00 & 1.07 \\
\hline Nervous disease & 1.10 & 1.07 & 1.13 \\
\hline Gastric disease & 0.77 & 0.75 & 0.79 \\
\hline Alcoholic liver disease & 1.87 & 1.66 & 2.09 \\
\hline Atherosclerosis & 1.05 & 1.02 & 1.09 \\
\hline Infections/parasites & 1.14 & 1.11 & 1.17 \\
\hline External injuries & 0.97 & 0.95 & 1.00 \\
\hline Smoking related cancers & 1.72 & 1.66 & 1.79 \\
\hline Non smoking related cancer & 1.32 & 1.27 & 1.37 \\
\hline
\end{tabular}

(see Table 2)

Source: AOK claims data, own calculations

\section{Abbreviations}

El: Extremity injury; LTC: Long-term care; SEl: Severe extremity injuries: NSEl: Non-severe extremity injuries

\section{Acknowledgements}

The dataset supporting the conclusions of this article is not publicly available. Data access was legally approved by the "Wissenschaftliches Institut der Ortskrankenkassen" (WIdO). The study is based on anonymised administrative claims data that never involved patients directly. Individual patients cannot be identified and the analyses presented do not affect patients whose anonymized records were used. This work was supported by the German Federal Ministry of Education and Research [grant number 16SV6373]. The sponsor did not interfere with the work in any way.

\section{Availability of data and materials}

The dataset supporting the conclusions of this article is not publicly available. Data access was legally approved by the "Wissenschaftliches Institut der Ortskrankenkassen (WIdO)" (Scientific Institute of Local Health Insurers).

\section{Authors' contributions}

$A B$ carried out the statistical analysis and drafted the manuscript. AV, $Y Z$ and $A V$ participated in the preparation of the data along with $A B$. GD and $A B$ conceived the study, AB implemented the study. All authors read and approved the final manuscript.

\section{Competing interests}

The authors declare that they have no competing interests.

\section{Consent for publication}

Not applicable.

\section{Ethics approval and consent to participate}

The study is based on anonymized administrative claims data that never involved patients directly. Individual patients cannot be identified and the analyses presented do not affect patients whose anonymized records were used. The patients were never directly involved in the study. Therefore, no ethical approval was neccessary and no consent to participte could be obtained.

\section{Author details}

${ }^{1}$ Empirical Social Research and Demography, Institute of Sociology and Demography, University of Rostock, Ulmenstr. 69, 18057 Rostock, Germany. ${ }^{2}$ German Center for Neurodegenerative Diseases, Rostock, Germany. ${ }^{3}$ Rostock Center for the Study of Demographic Change, Rostock, Germany. ${ }^{4}$ German Center for Neurodegenerative Diseases, Bonn/Rostock, Germany.

Received: 5 February 2016 Accepted: 23 November 2016

Published online: 06 December 2016

\section{References}

1. Brown CJ, Flood KL. Mobility limitation in the older patient. JAMA. 2013;310:1168.

2. Shumway-Cook A, Ciol MA, Yorkston KM, Hoffman JM, Chan L. Mobility limitations in the medicare population: prevalence and sociodemographic and clinical correlates. J Am Geriatr Soc. 2005;53:1217-21.

3. Fasano A, Plotnik M, Bove F, Berardelli A. The neurobiology of falls. Neurol Sci. 2012;33:1215-23.

4. Aksari P, Stoppe G. Risikofaktoren der Alzheimer-Demenz. Fortschritte der Neurologie Psychiatrie. 1996;64:425-32.

5. Luppa M, Riedel-Heller SG, Luck T, Wiese B, Bussche H, Haller F, et al. Age-related predictors of institutionalization: results of the German study on ageing, cognition and dementia in primary care patients (AgeCoDe). Soc Psychiatry Psychiatr Epidemiol. 2012;47:263-70.

6. Balzi D, Lauretani F, Barchielli A, Ferrucci L, Bandinelli S, Buiatti E, et al. Risk factors for disability in older persons over 3-year follow-up. Age Ageing. 2009;39:92-8.

7. Bouwen A, de Lepeleire J, Buntinx F. Rate of accidental falls in institutionalised older people with and without cognitive impairment halved as a result of a staff-oriented intervention. Age Ageing. 2008;37:306-10. 
8. Sauvaget C, Yamada M, Fujiwara S, Sasaki H, Mimori Y. Dementia as a predictor of functional disability: a four-year follow-up study. Gerontology. 2002:48:226-33.

9. Aschkenasy MT, Rothenhaus TC. Trauma and falls in the elderly. Emerg Med Clin North Am. 2006;24:413-32.

10. Kannus P, Niemi S, Parkkari J, Palvanen M, Heinonen A, Sievänen H, et al. Why is the age-standardized incidence of low-trauma fractures rising in many elderly populations? J Bone Miner Res. 2002;17:1363-67.

11. Doblhammer G. Demografie der Demenz. Bern: Verlag Hans Huber; 2012.

12. de Bruijn RF, Schrijvers EM, de Groot KA, Witteman JC, Hofman A, Franco $\mathrm{OH}$, Koudstaal PJ, Ikram MA, et al. The association between physical activity and dementia in an elderly population: the Rotterdam study. Eur J Epidemiol. 2013;28:277-83.

13. Hamer M, Chida Y. Physical activity and risk of neurodegenerative disease: a systematic review of prospective evidence. Psychol Med. 2009;39:3.

14. Zhou Y, Putter H, Doblhammer G. Years of life lost due to lower extremity injury in association with dementia, and care need: a 6-year follow-up population-based study using a multi-state approach among German elderly. BMC Geriatr. 2016;16:9.

15. Wang $\mathrm{H}$. Late-life engagement in social and leisure activities is associated with a decreased risk of dementia: a longitudinal study from the Kungsholmen Project. Am J Epidemiol. 2002;155:1081-87.

16. Lautenschlager NT, Cox KL, Flicker L, Foster JK, van Bockxmeer FM, Xiao J, et al. Effect of physical activity on cognitive function in older adults at risk for Alzheimer disease: a randomized trial. J Am Med Assoc. 2008;300:1027-37.

17. Aarsland D, Sardahaee FS, Anderssen S, Ballard C, the Alzheimer's Society Systematic. Is physical activity a potential preventive factor for vascular dementia? A systematic review. Aging Ment Health. 2010;14:386-95.

18. Fitzpatrick AL, Buchanan CK, Nahin RL, DeKosky ST, Atkinson HA, Carlson MC, et al. Associations of gait speed and other measures of physical function with cognition in a healthy cohort of elderly persons. J Gerontol. 2007;62A:1244-51.

19. Verghese J, Lipton RB, Hall CB, Kuslansky G, Katz MJ, Buschke H. Abnormality of gait as a predictor of non-Alzheimer's dementia. N Engl J Med. 2002;347: 1761-68.

20. Krogseth $M$, Wyller TB, Engedal K, Juliebø V. Delirium is an important predictor of incident dementia among elderly hip fracture patients. Dement Geriatr Cogn Disord. 2011;31:63-70.

21. Montero-Odasso M, Hachinski V. Preludes to brain failure: executive dysfunction and gait disturbances. Neurol Sci. 2014;35:601-04.

22. Nikolaus T. Gait, balance and falls - assessment and prevention. Dtsch Med Wochenschr. 2005;130:961-64.

23. Inagawa $T$, Hamagishi $T$, Takaso $Y$, Hitomi $Y$, Kambayashi $Y$, Hibino $Y$, et al. Decreased activity of daily living produced by the combination of Alzheimer's disease and lower limb fracture in elderly requiring nursing care. Environ Health Prev Med. 2013;18:16-23.

24. Doblhammer G, Fink A, Zylla S, Willekens F. Compression or expansion of dementia in Germany? An observational study of short-term trends in incidence and death rates of dementia between 2006/07 and 2009/10 based on German health insurance data. Alzheimers Res Ther. 2015;7:66.

25. Sherrington C, Fairhall N, Kirkham C, Clemson L, Howard K, Vogler C, et al. Exercise and fall prevention self-management to reduce mobility-related disability and falls after fall-related lower limb fracture in older people: protocol for the RESTORE (Recovery Exercises and STepping On afteR fracturE) randomised controlled trial. BMC Geriatr. 2016;16:34.

26. Osnes EK, Lofthus CM, Meyer HE, Falch JA, Nordsletten L, Cappelen I, et al. Consequences of hip fracture on activities of daily life and residential needs. Osteoporos Int. 2004;15:567-74.

27. Voukelatos A, Merom D, Sherrington C, Rissel C, Cumming RG, Lord SR. The impact of a home-based walking programme on falls in older people: the Easy Steps randomised controlled trial. Age Ageing. 2015;44:377-83.

28. Cameron ID, Gillespie LD, Robertson MC, Murray GR, Hill KD, Cumming RG, et al. Interventions for preventing falls in older people in care facilities and hospitals. Cochrane Database Syst Rev. 2012;12:CD005465.

29. Gillespie LD, Robertson MC, Gillespie WJ, Sherrington C, Gates S, Clemson LM, et al. Interventions for preventing falls in older people living in the community. Cochrane Database Syst Rev. 2012;9:CD007146.

30. Neyens JC, Dijcks BP, Twisk J, Schols JM, van Haastregt JC, van den Heuvel WJ, et al. A multifactorial intervention for the prevention of falls in psychogeriatric nursing home patients, a randomised controlled trial (RCT). Age Ageing. 2009;38:194-99.

31. Trombetti A, Hars M, Herrmann FR, Kressig RW, Ferrari S, Rizzoli R. Effect of music-based multitask training on gait, balance, and fall risk in elderly people: a randomized controlled trial. Arch Intern Med. 2011;171:525-33.

32. Agree EM, Freedman VA, Sengupta M. Factors influencing the use of mobility technology in community-based long-term care. J Aging Health. 2004;16:267-307.

33. Clarke $P$, Chan P, Santaguida PL, Colantonio A. The use of mobility devices among institutionalized older adults. J Aging Health. 2009;21:611-26.

34. Schulz R, Beach SR, Matthews JT, Courtney K, Devito Dabbs A, Person Mecca $L$, et al. Willingness to pay for quality of life technologies to enhance independent functioning among baby boomers and the elderly adults. Gerontologist. 2014;54:363-74.

35. Stenvall $M$, Berggren $M$, Lundström M, Gustafson $Y$, Olofsson B. A multidisciplinary intervention program improved the outcome after hip fracture for people with dementia-Subgroup analyses of a randomized controlled trial. Arch Gerontol Geriatr. 2012;54:e284.

\section{Submit your next manuscript to BioMed Central and we will help you at every step:}

- We accept pre-submission inquiries

- Our selector tool helps you to find the most relevant journal

- We provide round the clock customer support

- Convenient online submission

- Thorough peer review

- Inclusion in PubMed and all major indexing services

- Maximum visibility for your research

Submit your manuscript at www.biomedcentral.com/submit
C) Biomed Central 